\title{
Microstructure and Physical Properties of GaN Films on Sapphire Substrates
}

Zhizhong Chen, Rong Zhang, Jianming Zhu, Bo Shen, Yugang Zhou, Peng Chen, Weiping Li, Yi Shi, Shulin Gu and Youdou Zheng

National Laboratory of Solid State Microstructures and Department of Physics, Nanjing

University, Nanjing 210093, P.R.China

\begin{abstract}
Transmission electron microscopy (TEM), x-ray diffraction (XRD), photoluminescence (PL) and Raman scattering measurements were applied to study the correlation between the microstructure and physical properties of the GaN films grown by light radiation heating metalorganic chemical vapor deposition (LRH-MOCVD), using $\mathrm{GaN}$ buffer layer on sapphire substrates. When the density of the threading dislocation (TD) increases about one order of magnitude, the yellow luminescence (YL) intensity is strengthened from negligible to two orders of magnitude higher than the band edge emission intensity. The full width of half maximum (FWHM) of the GaN (0002) peak of the XRD rocking curve was widened from $11 \mathrm{~min}$ to $15 \mathrm{~min}$, and in Raman spectra, the width of $\mathrm{E}_{2}$ mode is broadened from $5 \mathrm{~cm}^{-1}$ to $7 \mathrm{~cm}^{-1}$. A "zippers" structure at the interface of $\mathrm{GaN} / \mathrm{sapphire}$ was observed by high-resolution electron microscope (HREM). Furthermore the origins of TD and relationship between physical properties and microstructures combining the growth conditions are discussed.
\end{abstract}

\section{INTRODUCTION}

$\mathrm{GaN}$ is a promising material for optoelectronic and electronic devices such as bluegreen light emitting diodes, laser diodes and high temperature, high frequency, high power transistors. Although blue current-injected laser diodes have been fabricated by $\mathrm{S}$. Nakamura [1], there are still many problems under debate. The origins of the YL emission remain unclear while it is commonly observed in almost all undoped and n-doped GaN epilayer grown by conventional methods [2-5]. The generation of TD in the epilayer of which density is high as $10^{8}-10^{10} \mathrm{~cm}^{-2}[6-8]$, and the nature and the role of initial nitridation of sapphire surface and the low-temperature growth GaN (AlN, InN) buffer layer which affects the GaN-based materials epitaxy growth [9-13] are ambiguous too. Several groups reported the relationships between GaN microstructures and XRD, PL, and Raman spectra, respectively [11,14-17]. But there are few works to try to resolve above problems by studying the microstructures and physical properties combining the growth conditions, such as the initial nitridation time and buffer layer thickness, and so on. In this paper, different properties of two kinds of GaN samples are grown by metalorganic chemical vapor deposition (MOCVD) under different conditions. Two sets of GaN films in TEM, XRD, PL and Raman measurements are investigated. According to the results, the origins of TD and YL are discussed.

F99W3.50 
Table 1 Growth conditions of the sample A and B

\begin{tabular}{l|cc}
\hline & Sample A & Sample B \\
\hline Nitridation time $(\mathrm{min})$ & 3 & 1.5 \\
Buffer growth temperature $\left({ }^{\circ} \mathrm{C}\right)$ & 500 & 500 \\
Buffer thickness $(\mathrm{nm})$ & 50 & 25 \\
Epilayer Growth temperature $\left({ }^{\circ} \mathrm{C}\right)$ & 950 & 950 \\
TMG flow $(\mu \mathrm{mol} / \mathrm{min})$ & 20 & 28 \\
$\mathrm{NH}_{3}$ flow $(1 / \mathrm{min})$ & 1.65 & 2.35 \\
Epilayer thickness $(\mu \mathrm{m})$ & 0.9 & 1.7 \\
\hline
\end{tabular}

\section{EXPERIMENTS}

The GaN films were grown by LRH-MOCVD on (0001) sapphire substrates. Details of the growth processes are given in Ref. 18. Table 1 shows the growth conditions of two typical samples which named as sample A and sample B.

Transmission electron microscope (high-resolution electron microscope) samples were prepared by the standard technique. Two pieces of GaN specimen were bonded by way of face-to-face by 504 glue. After mechanical grinding and polishing of the "sandwich" on the cross-sectional plane to a thickness of 20-30 $\mu \mathrm{m}$, the specimens were further thinned to electron transparency with an $\mathrm{Ar}^{+}$ion mill. TEM (HREM) observations were carried out with a JEOL JEM-4000EX microscopy operated at $400 \mathrm{kV}$.

\section{RESULTS AND DISCUSSION}

Figure 1 shows typical cross-sectional TEM images of two samples near the
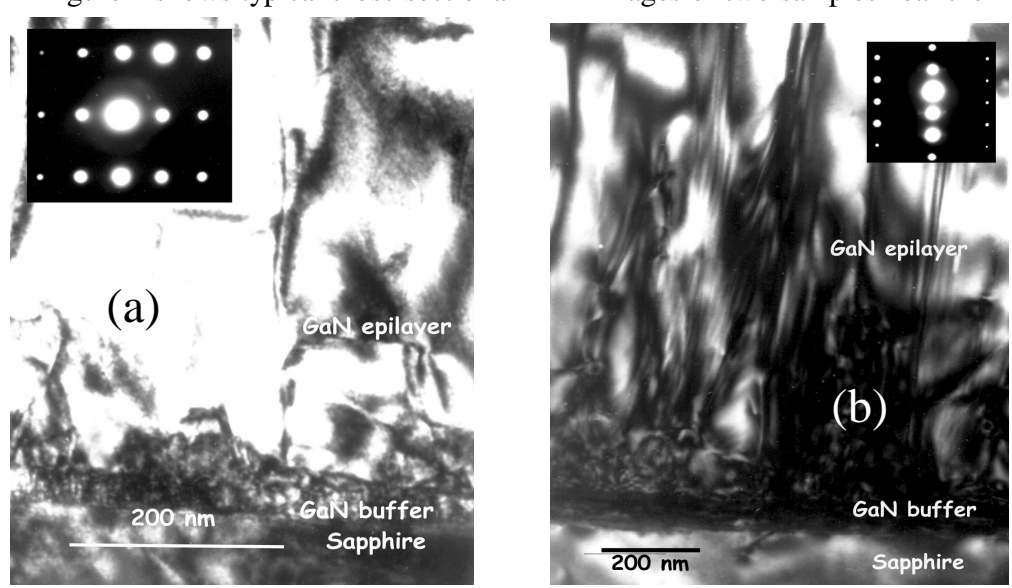

Figure 1. Cross-sectional TEM images of GaN/sapphire. The inserts are the electron diffraction (ED) patterns of the GaN epilayers. The density of threading dislocation in (a) is an order lower than that in (b). (a) and (b) are corresponding to the sample $A$ and $B$ 
GaN/sapphire interface. The inserts show the electron diffraction (ED) patterns of the $\mathrm{GaN}$ epilayers top regions. Corresponding to the growth processes, there are three zones in the GaN layer: buffer zone, "faulted" zone and "sound" zone. The buffer zone is above the interface between the GaN layer and the sapphire substrate, about $50 \mathrm{~nm}$ in figure 1(a) and $25 \mathrm{~nm}$ in figure $1(\mathrm{~b})$, which were grown at low temperature about $500^{\circ} \mathrm{C}$. The "faulted" zone is $0.4 \mu \mathrm{m}$ thick from the top of buffer layer into GaN epilayer. There are some "haystack-like" domains in the very beginning. Among these domains, there are high density of dislocations and other defects, and just above these domains, the dislocation density reduced sharply. The area above the "faulted" zone, which is called "sound" zone, is with very low density of defects, uniform and high quality which can be concluded from ED patterns. In comparison, the sample A shows about an order lower density of threading dislocation (TD) than the sample B, its columnar diameters is larger and its ED pattern is sharper, too. All these results indicate that the crystal quality of the sample A is higher than the sample B.

In order to study the buffer layer difference between the sample A and B, we performed HREM observation. Figure 2 shows the HREM of the interfaces of GaN/ $\alpha$ $\mathrm{Al}_{2} \mathrm{O}_{3}$ of the sample B. We find "zippers" structure at the $\mathrm{GaN} / \alpha-\mathrm{Al}_{2} \mathrm{O}_{3}$ interface. The gears of the zipper are perpendicular to the interface, and the period is about $1.8 \mathrm{~nm}$. There are more than one "zipper" standing side by side in some areas. The "zipper" zone fluctuates from $10 \mathrm{~nm}$ thick to $25 \mathrm{~nm}$. In HREM images of the sample A, haze and inhomogeneous contrast in GaN layer is observed among nearly $100 \mathrm{~nm}$ above the interface of $\mathrm{GaN} / \alpha-\mathrm{Al}_{2} \mathrm{O}_{3}$. It seems that crystal quality of buffer layer is poor. According to the study of the "zippers" structure in sample B [19], the amorphous-like growth predominated at the beginning of the buffer layer growth. So did in the sample A. It can be seen that in table 1 that the sample A was grown with longer nitridation time and thick buffer layer. Decreasing the density of nucleation centers by elongating the initial nitridation advantages to the lateral growth when epitaxy growth begins. To increase the thickness of the buffer layer can increase the thickness of amorphous zone which is beneficial to relax the strain. The layers are believed to be nearly free of extended defects due to the solid-phase crystallization process that take place during the heating step prior

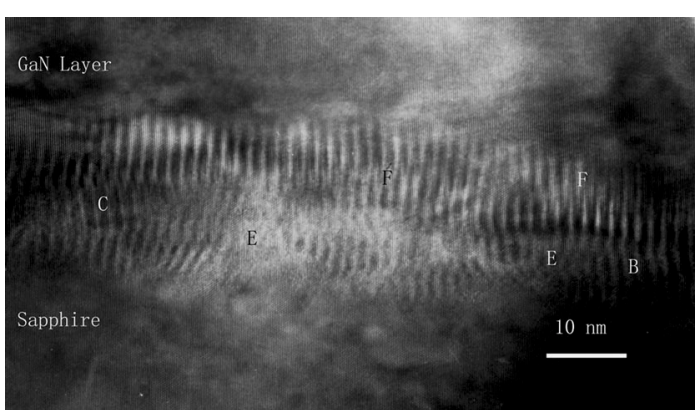

Figure 2. HREM images of the sample B. "Zipper" appears at the interface of $\mathrm{GaN} / \alpha-\mathrm{Al}_{2} \mathrm{O}_{3}$. The gears of the "zipper" period is $1.8 \mathrm{~nm}$ to $\mathrm{GaN}$ deposition at higher temperature [15]. The truncated hexagonal pyramidal mesas in the amorphous zone may grow larger, which is also advantageous to the lateral growth [10-11].

Figure 3 shows the x-ray diffraction spectra of the two epitaxial $\mathrm{GaN}$ films. The $\theta / 2 \theta$ scan of the GaN films on the basalplane sapphire shows dominant peaks corresponding to (0002)

peaks and their harmonic. The FWHM $\delta \theta$ of a Bragg peak in a $\theta / 2 \theta$ scan can be 


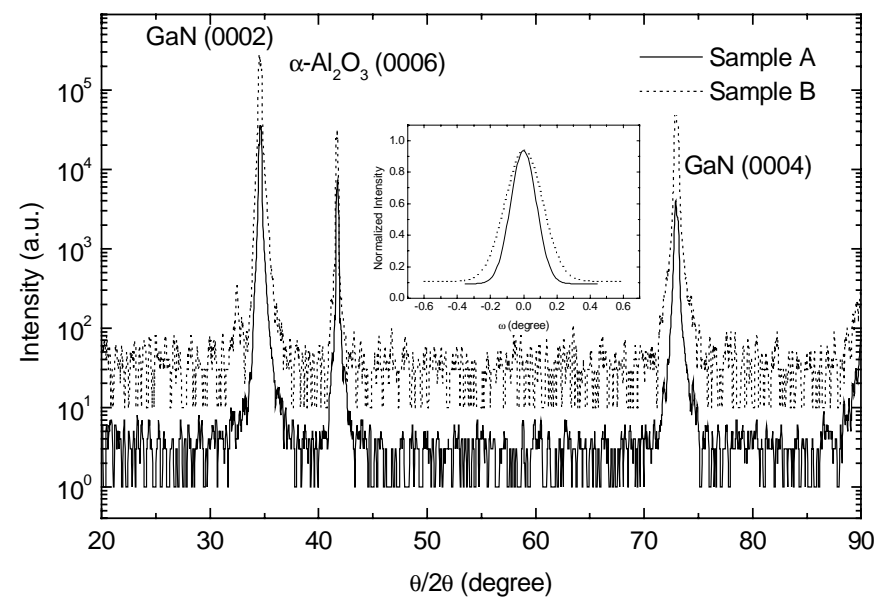

Figure 3. The $x$-ray diffraction spectra of sample $A$ and $B$. The average domain size and inhomogeneous strain of the sample $A$ and $B$ are calculated as 1753, $909 \AA$ and $0.167 \%, 0.141 \%$ by the width and the diffraction angle of (0002) and (0004) peaks The insert is XRD rocking curve of GaN epilayers of (0002) reflection. The FWHM of the sample $A$ and $B$ are 11 min and $15 \mathrm{~min}$, respectively.

expressed by the following equation [20] ,

$$
\delta \theta=\lambda /\left(2 D \cos \theta+\varepsilon_{\text {in }} \operatorname{tg} \theta\right)
$$

Here $\mathrm{D}$ is the average domain size, $\varepsilon_{\text {in }}$ is the inhomogeneous strain. The values of $\delta \theta$ of $\mathrm{GaN}$ (0002), (0004) peaks in figure. 3 are $0.056^{\circ}, 0.102^{\circ}$ for sample A and $0.069^{\circ}, 0.084^{\circ}$ for sample B. From Equation (1), (D, $\varepsilon$ in ) of sample A,B can be obtained $\left(1750^{\circ}\right.$, $0.167 \%),\left(900^{\circ}, 0.141 \%\right)$, respectively by the value of $\theta$ and $\delta \theta$. It is agreed to the results of TEM, in which the sample A columnar diameters is higher and its ED pattern is shaper. V. Potin et al. and Ning et al. think the TD is originated from the low angle domain boundaries $[6,8]$, which can be proved by our results. It seems that there are reactions of partial dislocations [7] since the $\varepsilon_{\text {in }}$ in sample B is lower in sample A. The insert in figure 3 shows the x-ray rocking curve of the two samples. The FWHM from (0002) reflections of the two samples are 11 and 15 minutes (min), respectively. The FWHM of the sample A is 4 min lower than that of the sample B. This result may be due to the smaller average tilts of column crystallites in the epilayer of the sample A. If the caxis is slightly tilted, screw dislocation with $\mathbf{b}=[0001]$ will be present [6]. What the c-axis screw TD and mixed TD broaden the FWHM of (0002) peak of XRD rocking curve is clarified by B. Heying et al. [16]. As reported by S. Christiansen et al. [21], the YL of $\mathrm{GaN}$ was associated with c-axis screw dislocations. We prefer the broadened FWHM due to screw TD after referring to the following photoluminescence (PL) measurement.

Room temperature PL spectra of GaN epilayers are shown in figure 4. Both spectra have three characteristic regions: band-edge and donor-acceptor pair emission (3.2-3.5 $\mathrm{eV}$ ) which are not easy to be distinguished at room temperature, blue band emission (2.7- 
$3.2 \mathrm{eV}$ ) and yellow band emission (1.9-2.5 eV). However, different samples have different types of spectra. The intensity ratio of the YL to the band-edge emission of the sample B is 3 orders higher than that of the sample A. Furthermore the YL of the sample B is stronger and has fine structure with two peaks at 2.10 $\mathrm{eV}$ and $2.29 \mathrm{eV}$, while that of the sample A is nearly invisible in the PL spectrum. As reported by F.A. Ponce [4], the intensity of the near bandedge PL emission of $\mathrm{GaN}$ is indicative of its optical quality. So the sample A has a higher optical quality than the sample B, which corresponds the high crystal quality concluded by the above TEM experiment. The YL intensity can vary over a wide range to $\mathrm{GaN}$ epilayers grown with different conditions. The GaN epilayers with high quality exhibits almost no YL emission. Although the
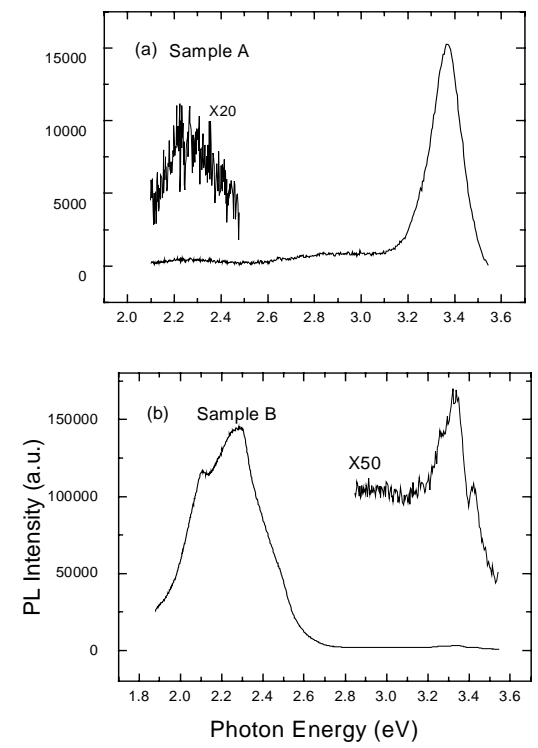

Figure 4. Photoluminescence of GaN epilayers, the intensity ratio of YL to band-edge emission in (b) is 3 orders of that in (a). There are fine origin of the $\mathrm{YL}$ is still being debated, it is well acknowledged that the YL corresponds to the deformed crystal structure [5]. In our work, it seems that the YL is related to the screw TD. The growth condition may be concerned for the YL origin [5].

The Raman Spectra for the two GaN samples are measured. According to Ref.17, in the sample A, 417 and $568 \mathrm{~cm}^{-1}$ are assigned as $A_{1 \mathrm{~g}}$ mode of sapphire and $\mathrm{E}_{2}$ mode of $\mathrm{GaN}$. The sample $\mathrm{B}$ is assigned in the same way. In contrast to the $\mathrm{E}_{2}$ mode of the sample A whose width is $5 \mathrm{~cm}^{-1}$, that of the sample B is widened to $7 \mathrm{~cm}^{-1}$, which means that in the sample $\mathrm{B} \mathrm{E}_{2}$ mode has shorter lifetime. It also shows that the sample $\mathrm{B}$ must be less perfect of crystal quality than the sample A.

\section{CONCLUSIONS}

The crystalline structure of GaN epilayers has been studied using TEM and double crystal x-ray diffraction. When the density of TD increases about an order of magnitude, the FWHM of $\mathrm{GaN}$ (0002) peak of x-ray diffraction rocking curve is increased from 11 $\min$ to $15 \mathrm{~min}$. The YL intensity is strengthened from negligible to two orders of bandedge emission intensity. The "zippers" structure is observed by HREM measurement of the $\mathrm{GaN} / \alpha-\mathrm{Al}_{2} \mathrm{O}_{3}$ interface. From the above results, we concluded the high quality GaN epilayer can be grown by optimum initial nitridation time and buffer layer thickness.

\section{Acknowledgement}

National High Technology Research and Development Project of China and 
National Natural Science Foundation of China (contracts \#69976014, \#69636010, \#69806006, \#69987001) support this work.

\section{REFERENCES}

1. S. Nakamura et al., Jpn. J. Appl. Phys., 35, L74 (1996).

2. T. Ogino and M. Aoki, Jpn. J. Appl. Phys. 19, 295 (1998).

3. J. Neugebauer and C.G. Van de Walle, Appl. Phys Lett., 69, 503 (1996).

4. F.A. Ponce, D.P. Bour, W. Götz and P.J. Wright, Appl. Phys. Lett., 68, 57 (1996).

5. $\quad$ R. Zhang and T.F. Kuech, Appl. Phys. Lett., 72, 1611 (1998).

6. X.J. Ning, F.R. Chien, P. Pirous, J.W. Yang and M. A. Khan, J. Mater. Res., 11, 580 (1996).

7. M.S. Hao, T. Sugahara, H. Sato, Y. Morishima, Y. Naoi, L.T. Romano, S. Sakai, Jpn. J. Appl. Phys. 37, part2 (3A), L291 (1998).

8 V. Potin, P. Vermaut, P. Ruterana and G. Nouet, J. Electron. Mater.,27, 266 (1998).

9. S. Nakamura, Jpn. J. Appl. Phys. 30, L1705 (1991).

10. I. Akasaki, H. Amano, Y. Koide, K. Hiramatsu and N. Sawaki, J. Crystal Growth 98, 209 (1989).

11. G. Li, S.J. Chua, S.J. Xu, W. Wang, P. Li, G. Beaumont, and P. Gibart, Appl. Phys. Lett. 74, 2821 (1999).

12. N. Grandjean, J. Massies, and M. Leroux, Appl. Phys. Lett. 69, 2071 (1996).

13. S. Fuke, H. Teshigawara, K. Kuwahara Y. Takano, T. Ito, M. Yanagihara, K. Ohtsaka, J. Appl.Phys. 83, 764 (1998).

14. S.J. Rosner, E.C. Carr, M.J. Ludowise, Appl. Phys. Lett., 70, 420 (1997).

15. F.A. Ponce, B.S. Krusor, J.S. Major, M.E. Plano, and D.F. Welch, Appl .Phys. Lett, 67(3), 410 (1995).

16. B. Heying, X.H. Wu, S. Keller, Y. Li, D. Kapolnek, B.P. Keller, S.P. DenBaars, and J.S. Speck, Appl Phys. Lett. 68(5), 643 (1996).

17. S.-C.Y. Tsen, D. J. Smith, K.T. Tsen, W. Kim and H. Morkoc, J. Appl. Phys. 82, 6008 (1997).

18. B. Shen, Y.G. Zhou, Z.Z. Chen, P. Chen, W.P. Li, Y.D. Zheng, Appl. Phys. A 68, 593 (1999).

18. Z.Z. Chen, J.M. Zhu, R. Zhang B. Shen,, Y.G. Zhou, P. Chen, W.P. Li (unpublished).

20. R.W. Vook, in Epilayer Growth, edited by J.W. Matthews, (Academic, New York, 1975), p.339.

21. S. Christiansen, M. Albrecht, W. Dorsch, Mater. Sci. Eng. B43, 296 (1997). 